\title{
Spinal manipulation in the treatment of patients with MRI-confirmed lumbar disc herniation and sacroiliac joint hypomobility: a quasi-experimental study
}

\author{
Esmaeil Shokri ${ }^{1}$, Fahimeh Kamali ${ }^{1,2,3^{*}}$, Ehsan Sinaei ${ }^{2}$ and Farahnaz Ghafarinejad ${ }^{1,2}$
}

\begin{abstract}
Background: To investigate the effect of lumbar and sacroiliac joint (SIJ) manipulation on pain and functional disability in patients with lumbar disc herniation (LDH) concomitant with SIJ hypomobility.

Methods: Twenty patients aged between 20 and 50 years with MRI-confirmed LDH who also had SIJ hypomobility participated in the trial in 2010. Patients who had sequestrated disc herniation were excluded. All patients received five sessions of spinal manipulative therapy (SMT) for the SIJ and lumbar spine during a 2-week period. Back and leg pain intensity and functional disability level were measured with a numerical rating scale (NRS) and the Oswestry Disability Index (ODI) at baseline, immediately after the 5th session, and 1 month after baseline.

Results: A significantly greater mean improvement in back and leg pain was observed in the 5 th sessions and 1 month after SMT. Mean changes in ODI in the 5th session and 1 month after treatment also showed significant improvement. The MCIC for NRS and ODI scores in the present study were considered 20 and 6 points, respectively. Therefore, the mentioned improvements were not clinically significant in the 5th session or at 1-month follow-up. Conclusion: Five sessions of lumbar and SIJ manipulation can potentially improve pain and functional disability in patients with MRI-confirmed LDH and concomitant SIJ hypomobility.
\end{abstract}

Trial registration: Irct.ir (Identifier: IRCT2017011924149N33), registered 19 February 2017 (retrospectively registered).

Keywords: Spinal manipulation, Lumbar disc herniation, Sacroiliac joint, Back pain

\section{Background}

Common low back problems include disc prolapse, spinal stenosis and low back pain [1]. Disc herniation can be categorized as protrusion (disc contained by the annulus fibrosus), extrusion (disc materials migrated out through the annulus fibrosus, but contained by the posterior longitudinal ligament) and sequestration (disc materials released into the spinal canal) [1]. Disc prolapse commonly presents with pain and numbness radiating to the buttocks and legs due to spinal nerve or nerve

\footnotetext{
*Correspondence: fahimekamalii@gmail.com

${ }^{1}$ Physical Therapy Department, School of Rehabilitation Sciences, Shiraz University of Medical Sciences, Shiraz, Iran

${ }^{2}$ Rehabilitation Sciences Research Center, Shiraz University of Medical

Sciences, Shiraz, Iran

Full list of author information is available at the end of the article
}

root compromise; however, it may be asymptomatic in approximately $24 \%$ of all cases [1]. Symptomatic lumbar disc disease (SLDD) is a term used to differentiate between structural abnormalities without clinical symptoms and abnormalities that induce clinical presentations [2]. Approximately $95 \%$ of all instances of lumbar disc herniation (LDH) occur at L4-L5 and L5-S1 levels [1].

Lumbar disc herniation commonly presents with low back pain, and this problem is usually associated with sacroiliac joint (SIJ) disorders. In fact, up to $30.7 \%$ of patients with LBP and sciatica also have SIJ dysfunction [3]. A recent study reported the prevalence of SIJ dysfunction as $72.3 \%$ among patients with LDH [4]. Researchers have claimed that depending on the type of SIJ disorder, the lumbar spine (mostly L5) can also be 
involved [5]. The SIJ is part of the lumbar- pelvic-hip complex; since this complex works as a mechanical unit, the involvement of any structure can affect the position and movement of other sections [6].

Generally speaking, most patients with SLDD prefer conservative treatments to surgical intervention. To date, evidence has supported several conservative treatments for SLDD including traction [7], McKenzie extension exercises [8] and rehabilitation [9].

The use of spinal manipulative therapy (SMT) for patients with SLDD has also been suggested; however, its safety and indications have remained debatable, particularly in individuals with disc disruption or instability [2]. In this regard, the risk of SMT causing clinically worsened disc herniation or cauda equine syndrome in patients with LDH is estimated to be less than one in 3.7 million [2]. A systematic review in 2004 also confirmed the safety and effectiveness of SMT for patients with SLDD [2].

Some studies reported significant clinical improvements in patients with SLDD after manipulation in comparison to traction [10], heat [11] and sham manipulation [12], but no significant differences when compared to exercise therapy and medical corsets [11]. A recent study found long-term improvement in pain and functional activity after 1 year of follow-up [13], and another study in 2016 reported significant improvement in leg pain after 1 month in patients with extrusion and sequestration of lumbar discs, following manipulation [14].

Sacroiliac joint hypomobility has usually been overlooked in the management of patients with LDH and low back problems. However, there is no conclusive evidence for the effectiveness of SMT in the treatment of patients with $\mathrm{LDH}$, and the evidence to date is contradictory. Therefore, the present study aimed to investigate the effect of SMT applied to the lumbar spine and SIJ to treat patients with SLDD who also had SIJ hypomobility.

\section{Methods}

Participants

Twenty patients ( 11 males, 9 females) aged $20-50$ years old with MRI-proven unilateral LDH were included IN 2010 if they had SLDD in the L4-L5 or L5-S1 segment concomitant with ipsilateral SIJ hypomobility (Table 1). Leg pain during 1 to 10 months before the study was their major complaint, and the mean level of leg pain during the previous $24 \mathrm{~h}$ was $30-70$ out of 100 on a 0 100 numerical rating scale (NRS). The time interval allowed between the MRI diagnosis and inclusion in the study was 3 months. Exclusion criteria were sequestrated LDH with neurological signs, spinal canal stenosis, spondylolisthesis, previous lumbar surgery and gross instability. Patients a with positive well straight leg raise (SLR) test, indicating rather large disc herniation and poor
Table 1 Participants' demographic characteristics $(N=20)$

\begin{tabular}{lll}
\hline Variable & & Value $^{a}$ \\
\hline Age & & $37.86 \pm 9.62$ \\
BMI & Right & $25.10 \pm 3.12$ \\
Side of LDH & Left & $11(55)$ \\
& Right & $9(45)$ \\
Side of SIJ hypomobility & Left & $8(40)$ \\
& L4 - L5 & $12(60)$ \\
Segment of LDH & L5 - S1 & $5(25)$ \\
\hline
\end{tabular}

Abbreviations: $B M I$ body mass index, $L D H$ lumbar disc herniation, $S I J$ sacroiliac joint

${ }^{a}$ Values are mean \pm SD for continuous variables and number (percentage) for categorical variables

prognosis for conservative treatments $[15,16]$, were also excluded.

\section{Study design}

This was a pre-post test quasi-experimental study. The participants were selected among patients referred to physical therapy clinics of Shiraz, Iran. Sample size was calculated based on the NRS pain score reported in a previous related study (mean $[95 \% \mathrm{CI}]=22$ [15-30], $\alpha=$ $0.05, \beta=0.02$ ) [17]. Written informed consent was obtained and ethical approval was granted by the Shiraz University of Medical Sciences Ethics Committee (approval number CT-88-4614).

Demographic data, pain intensity, functional disability and clinical diagnostic tests were recorded at baseline. After that, the patients received five sessions of manipulative therapy on alternate days, and the outcomes were reassessed after the 1st and 5th sessions and at a 1month follow-up. All patients received both lumbar and SIJ manipulations in each treatment session.

\section{Interventions \\ Lumbar rotation manipulation}

The neutral position of the spine was used for sideposture lumbar manipulation. The patient lay on the asymptomatic side (e.g. left) in the lateral recumbent position, with his or her upper foot in the popliteal fossa of the lower leg. Standing opposite to the patient, the therapist grasped the patient's lower shoulder and arm and applied right rotation until motion was felt in the desired segment of the lumbar spine. The patient was rolled toward the therapist, with his or her arms positioned around the therapist's right arm. The therapist's right forearm was in contact with the patient's right axilla and pectoral region to maintain appropriate rotation from above. In this position, the therapist applied a high-velocity low-amplitude thrust to the pelvis in an anterior direction with his or her left forearm placed 
behind the patient's right hip. The therapist pressed the spinous process of the upper vertebra downward with his or her right thumb, while pulling the spinous process of the lower vertebra upward with his or her left thumb. The procedure was done in a way that avoided exacerbating the patient's pain at the barrier point [18] (Fig. 1).

\section{Sacroiliac joint manipulation}

The patients lay supine on a treatment table, with their fingers interlocked behind their head. The therapist stood contralateral to the side to be manipulated and moved the patient onto his or her side, then leaned toward the dysfunction side, rotated the patient, and exerted a quick thrust to the anterior superior iliac spine in the posterior and inferior directions [18, 19] (Fig. 2).

\section{Outcome measures}

The scores for back and leg pain were recorded separately on an NRS of 0 to 100, in which 0 indicated no pain and 100 indicated the worst perceived pain [20]. The minimal clinically important change (MCIC) in NRS score was 20 points [21, 22].

The patients' functional disability level was determined with the Oswestry Disability Index (ODI), a 10-item questionnaire in which each item is scored from 0 to 5 [23]. The maximum score on the ODI is 50 , and higher scores indicate greater functional disability. However, in the present study the maximum score was 45 , since the sex life item was omitted due to cultural issues. Therefore relative values are reported here as the total score/ total possible score $\times 10$. The MCIC for the ODI was reported as 6 points in a sample of patients with LBP who received physical therapy [24].

Participants were also assessed with the SLR and slump tests to diagnose LDH, and standing flexion, sitting flexion and Gillet tests were used to diagnose SIJ hypomobility. Although the evidence is contradictory, some studies have reported acceptable reliability for the SI) test. [25-27] The results of five clinical tests were

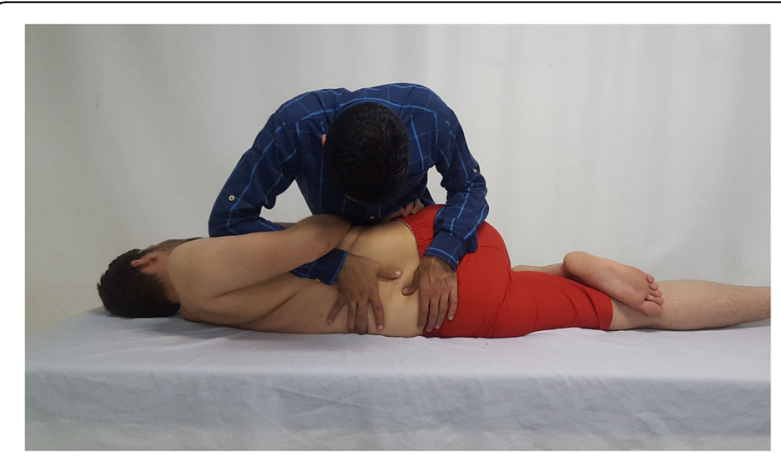

Fig. 1 Lumbar rotation manipulation

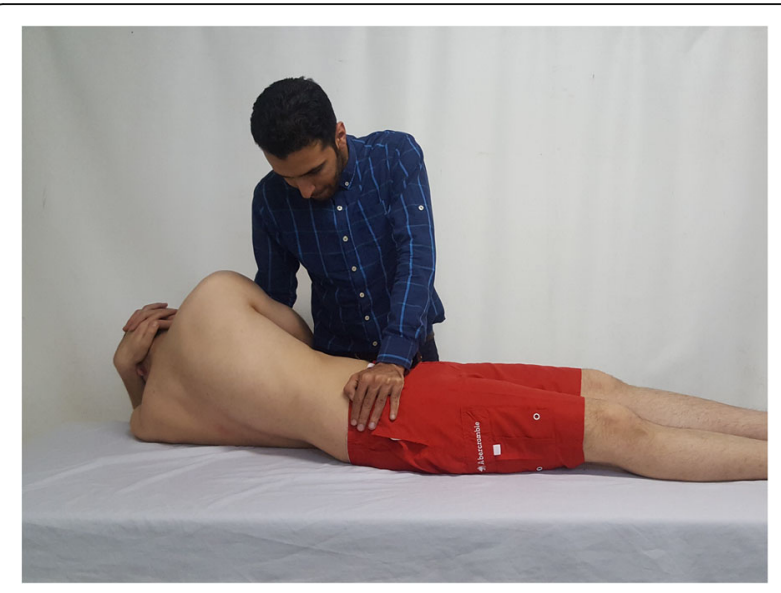

Fig. 2 Sacroiliac joint manipulation

recorded as positive or negative values at baseline, in the 5th session and 1 month after baseline.

\section{Statistical analysis}

The data were analyzed with the Statistical Package for Social Sciences (SPSS), version 21.0 (IBM Corp., Armonk, NY, USA). The Kolmogorov-Smirnov test of normality was conducted for all quantitative variables. Repeated measure ANOVA was used to assess the trends in changes in the NRS and ODI scores. Individual time point differences were determined by the Bonferroni post hoc test, and the results of the five clinical tests were analyzed with the McNemar test.

\section{Results}

The NRS score for back pain showed statistically significant improvement in the 5th session $(P=0.034)$ and at 1-month follow-up $(P=0.047)$ compared to the baseline value. In addition, statistically significant improvement in the leg NRS score was seen in the 5th session $(P=0$. $010)$ and at 1 -month follow-up $(P=0.006)$. Because the MCIC for NRS scores in the present study was 20 points, NRS score improvements in back and leg pain were not clinically significant in the 5th session or at 1month follow-up (Figs. 3 and 4) (Table 2).

The ODI scores indicated statistically significant improvement in the 5 th session $(P=0.001)$ and at 1 -month follow-up $(P=0.001)$. Because the MCIC for ODI score in the present study was 6 points, the improvements were not considered clinically significant at the 5th session or at 1-month follow-up (Fig. 5) (Table 2).

In $78.3 \%$ of our patients, the sides of SIJ hypomobility and LDH were the same. After treatment, the patients showed statistically significant improvements in Gillet, standing flexion and sitting flexion tests in the 5th session and at 1 -month follow-up $(P \leq 0.001)$. The findings also indicated $95 \%$ improvement in the results of the SI) 


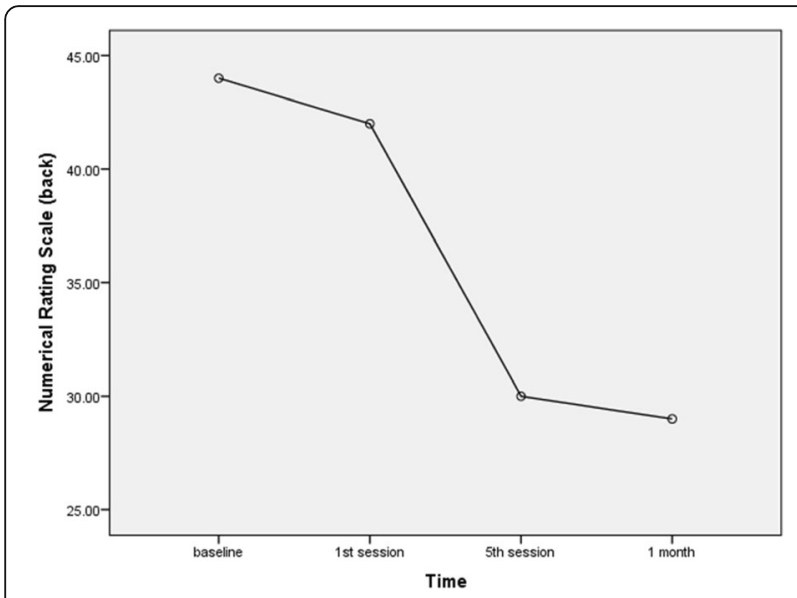

Fig. 3 Trend in back pain intensity during the trial

hypomobility tests after SMT. We also observed a $20 \%$ improvement in the SLR test results and a 15\% improvement in the slump test in the 5th session and at 1month follow-up after SMT, although these changes were not statistically significant (Table 3 ).

\section{Discussion}

The aim of the present study was to investigate the effect of SMT on pain, functional disability and the results of clinical tests of SIJ function and LDH in patients with unilateral SLDD plus SIJ hypomobility. Our findings suggest that five sessions of lumbar and SIJ manipulation can lead to statistically significant improvement in pain and functional disability, which in turn may restore normal SIJ mobility in these patients.

Compared to common treatments for LDH, SMT is reported to be 37,000 to 148,000 times safer than nonsteroid antiinflammatory drugs and 55,500 to 444,000 times safer than surgery [28]. Neither worsening of symptoms nor cauda equine syndrome were observed in

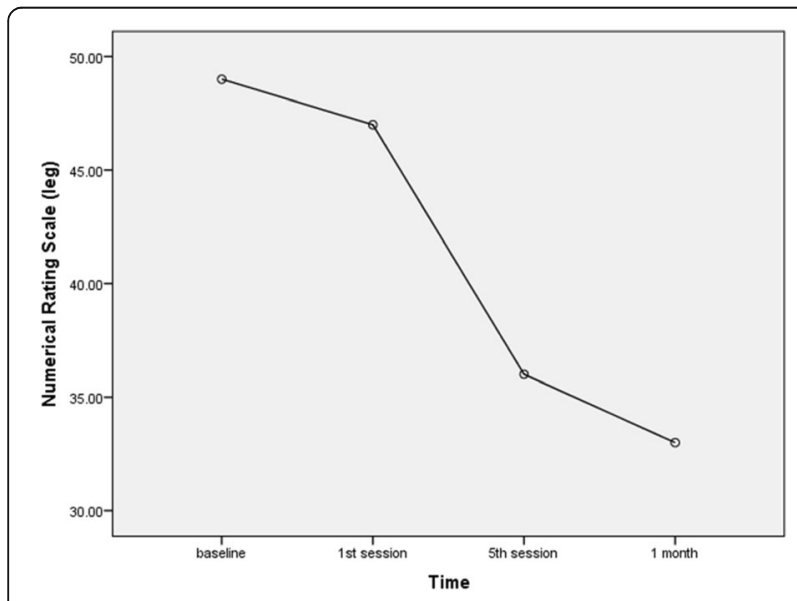

Fig. 4 Trend in leg pain intensity during the trial our participants after SMT. Epidemiologic data on the rate of injuries caused by manipulation are limited. The most common incidents are related to innocuous physiologic reactions or short-term discomfort generated at the treatment site. However, these are self-limiting events that usually resolve within $24 \mathrm{~h}$ after SMT [28].

In rotational side-posture lumbar manipulation, the impact of the facet joints limits axial rotation of the lower lumbar vertebrae and consequently prevents annulus fibrosus tearing [28]. In the present study, patients with sequestrated LDH who had neurological signs were excluded because these patients may have bowel and bladder disorders, and many (but not all) of them are thus candidates for surgery [29]. In the present study manipulation was applied in the neutral flexion-extension position to reduce the risk of injury.

The diagnosis and treatment of SIJ hypomobility in patients with SLDD are important issues that have not been adequately addressed in the literature. In $78.3 \%$ of our cases, the side of SIJ hypomobility was the same as the side of LDH. After treatment, 95\% improvement was obtained in the results of SIJ hypomobility tests (Table 2).

The SIJ has been reported to be one of the main sources of low back disorders [5]. A recent study also found that SI) dysfunction was a prevalent concomitant pathology in patients with LDH. Therefore, SIJ dysfunction should be considered in the treatment of these patients [4]. Pelvic asymmetry as well as hypermobility or hypomobility of the spinal or sacroiliac joints can cause low back pain $[3,30]$. Any involvement of the SIJ can induce muscle spasm in the piriformis, which in turn can lead to sciatic irritation and a wide range of symptoms mimicking radiculopathy [5]. Increased tension in the quadratus lumborum, iliopsoas or hamstring muscles may also affect the SIJ mechanism of action. Presumably, SIJ manipulation can decrease tension in these muscles and consequently correct lumbar spine dysfunction [3, 31].

Several mechanisms have been theorized for the mechanical and neurophysiological basis of SMT, including stimulation or modulation of the somatosensory system to evoke neuromuscular reflexes [32]. Forceful stretching of the spinal muscles induces relaxation after SMT. Other mechanisms are induced hypoalgesia [33], kinematic correction $[34,35]$ and increased lumbar range of motion [36]. A brief reduction in intradiscal pressure during SMT in cadavers and return to baseline within less than $1 \mathrm{~min}$ was reported in one earlier study [37]. Another study showed reduced H-reflex amplitude in patients with unilateral disc herniation, which improved after SMT [11]. The improved outcomes in our patients can be attributed to two main factors. Firstly, SIJ manipulation may improve normal functioning of the lumbar spine and related muscles [38]. Secondly, lumbar side-posture rotational manipulation can induce spinal muscle relaxation [32], 
Table 2 Mean values of back pain, leg pain and functional disability during the trial

\begin{tabular}{|c|c|c|c|c|c|}
\hline Outcomes & Baseline $^{a}$ & 1st session & 5th session & 1 month & Times with statistically significant differences ( $P$-value) \\
\hline \multirow[t]{2}{*}{ Back NRS } & $44.95 \pm 26.18$ & $42.00 \pm 25.30$ & $30.50 \pm 19.32$ & $29.75 \pm 16.42$ & Baseline - 5th session (0.034) \\
\hline & & & & & Baseline - 1 month (0.047) \\
\hline \multirow[t]{2}{*}{ Leg NRS } & $49.50 \pm 23.94$ & $47.25 \pm 24.35$ & $36.00 \pm 16.90$ & $33.75 \pm 13.75$ & Baseline -5 th session $(0.010)$ \\
\hline & & & & & Baseline - 1 month (0.006) \\
\hline \multirow[t]{2}{*}{ ODI } & $14.45 \pm 4.40$ & n.d. & $11.35 \pm 4.54$ & $10.95 \pm 4.27$ & Baseline -5 th session (0.001) \\
\hline & & & & & Baseline - 1 month (0.001) \\
\hline
\end{tabular}

Abbreviations: NRS numerical rating scale, ODI Oswestry Disability Index, n.d. not determined

a Values are mean $\pm S D$

improve lumbar range of motion [36], and briefly decrease intradiscal pressure [37].

The results of the SIJ hypomobility tests (including the Gillet test, standing flexion and sitting flexion tests) improved significantly in the 5 th session and after 1 -month follow-up compared to baseline values, whereas no statistically significant improvement was observed in the SLR and slump tests. Spinal manipulative therapy may enhance mobility of the SIJ and lumbar vertebrae, and affect the muscles in these regions, thus accounting for the improvement in pain and functional ability. However, significant changes in the slump and SLR tests may require additional interventions such as soft tissue manipulation and nerve mobilization, which were not tested in this study.

In one controlled trial, SMT and sham manipulation were compared in 102 participants with MRI-confirmed LDH; the SMT group showed significantly greater improvement in back and leg pain after 6 months [12]. Nevertheless, the intervention in that study was a combination of soft tissue manipulation and thrust manipulation, and the diagnosis and treatment of SIJ hypomobility were not considered.

In a prospective cohort study, Leemann et al., investigated the effect of high-velocity, low-amplitude SMT in

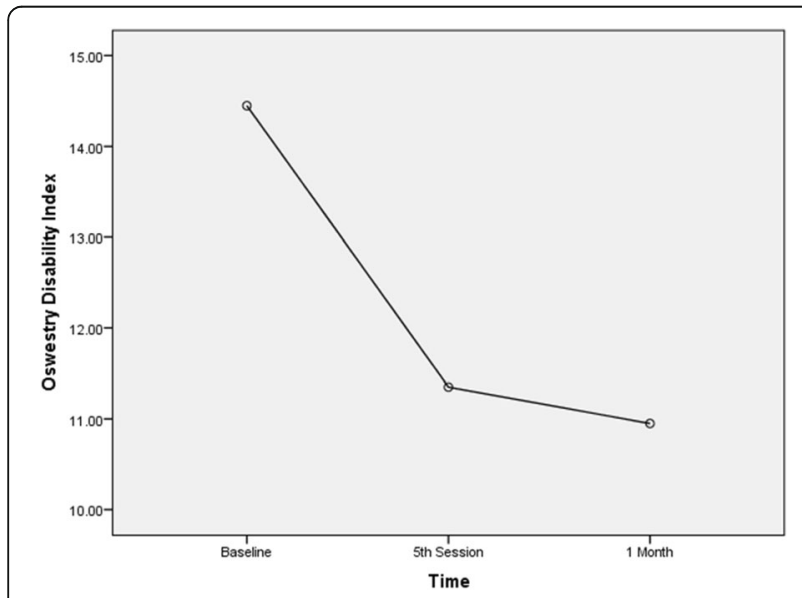

Fig. 5 Trend in functional disability level during the trial patients with acute or chronic MRI-confirmed LDH, and reported clinically significant improvement in back and leg NRS and ODI scores in both short-term and longterm assessments [13]. In a follow-up study, Ehrler et al., investigated the association of magnetic resonance imaging features, including axial location and type of herniation, with the outcomes of SMT in patients with LDH [14]. This study reported greater improvements in symptoms among patients with sequestrated SLDD who received SMT to the level of herniation. These studies, however, did not consider the treatment of the SIJ in patients with LDH.

Burton et al., also compared SMT with chemonucleolysis in the treatment of patients with SLDD, and reported greater improvements in back pain and disability in the first few weeks in the SMT group [39]. Their SMT, however, included a combination of thrust manipulation, mobilization and soft tissue stretching.

The results of previous studies have shown that SMT is effective in the treatment of LDH [40]. The study most similar to ours is the one by Galm et al., which included 150 patients with LDH, 46 of whom had SIJ dysfunction. All participants received routine physiotherapy, mobilization and SMT in the prone position. Significant improvements were reported in lumbar and ischiatic pain in the SIJ dysfunction group. These authors concluded that in the presence of lumbar and ischiatic symptoms, appropriate treatment for SIJ dysfunction should be considered regardless of intervertebral disc

Table 3 Distribution of improvements in diagnostic test results in the 5th session and after 1-month follow-up

\begin{tabular}{|c|c|c|c|c|}
\hline \multirow[t]{2}{*}{ Test } & \multicolumn{2}{|c|}{ 5th session } & \multicolumn{2}{|l|}{1 month } \\
\hline & $\begin{array}{l}\text { (Positive/ } \\
\text { Negative) }\end{array}$ & $P$-value & $\begin{array}{l}\text { (Positive/ } \\
\text { Negative) }\end{array}$ & $P$-value \\
\hline Gillet & $1 / 19$ & $<0.001^{*}$ & $1 / 19$ & $<0.001^{*}$ \\
\hline Standing flexion & $1 / 19$ & $<0.001^{*}$ & $1 / 19$ & $<0.001^{*}$ \\
\hline Sitting flexion & $1 / 19$ & $<0.001^{*}$ & $1 / 19$ & $<0.001^{*}$ \\
\hline SLR & $16 / 4$ & 0.12 & $17 / 3$ & 0.25 \\
\hline Slump & $17 / 3$ & 0.25 & $17 / 3$ & 0.25 \\
\hline
\end{tabular}

Abbreviations: SLR straight leg raising

${ }^{*}$ Significant recovery compared to baseline 
pathomorphology [3]. In this study, however, the number of treatment sessions and the results of SIJ and LDH physical tests were not reported.

Our study had some limitations which should be noted. The pre-post test design did not include a control group; consequently, the results cannot be considered evidence in support of the clinical efficacy of SMT for patients with LDH and SIJ hypomobility. A controlled trial is advisable in which combined manipulations are compared to lumbar or SIJ manipulation separately, to elucidate whether using both lumbar and SIJ manipulation together yields better outcomes than using a single type of manipulation. The small sample size and lack of long-term follow-up are other limitations. In addition, we are aware that measuring physiologic responses to SMT by recording electromyographic activity of the spinal muscles, the myotomes and dermatomes of the involved nerve roots, would strengthen the results of future studies. Also, more reliable tests for SIJ dysfunction are available and should be used in future studies. Despite these limitations, we addressed some shortcomings of previous studies. The strengths of the present study were matching of the physical examination findings with imaging findings, considering SIJ hypomobility in the treatment of patients with LDH, and the application of spinal thrust manipulation alone rather than a combination of therapeutic methods.

\section{Conclusions}

Spinal manipulative therapy can potentially improve pain, functional disability and SIJ mobility in patients with LDH concomitant with SIJ hypomobility; therefore, it can be implemented in physical therapy programs for these patients. However, further studies with larger sample sizes, longer follow-up periods and real control groups should be done to provide more accurate results.

\section{Abbreviations \\ LDH: Lumbar disc herniation; MCIC: Minimally clinically important change; NRS: Numerical rating scale; ODI: Oswestry disability index; SIJ: Sacroiliac joint; SLDD: Symptomatic lumbar disc disease; SMT: Spinal manipulative therapy}

\section{Acknowledgements \\ The authors would like to thank the Research Consultation Center (RCC) of Shiraz University of Medical Sciences for their assistance with English-language editing of this article, and K. Shashok (AuthorAID in the Eastern Mediterranean) for improving the use of English in the revised manuscript.}

Availability of data and materials

Please contact the corresponding author for raw data requests.

\begin{abstract}
Authors' contributions
Concept development and design: ESh, FK, FG. Supervision: FK, FG. Data collection/processing: ESh, FK, ES. Analysis/Interpretation: ESh, FK, ES. Writing and critical review: ESh, ES. All authors read and approved the final manuscript.
\end{abstract}

Ethics approval and consent to participate

Approval was granted by the Shiraz University of Medical Sciences Ethics Committee (approval code: CT-88-4614).

\section{Consent for publication}

Written informed consent was obtained from the patients for publication of their individual details and accompanying images in this manuscript. The consent form is held by the authors and is available for review by the Editor-in-Chief.

\section{Competing interests}

The authors declare that they have no competing interests.

\section{Publisher's Note}

Springer Nature remains neutral with regard to jurisdictional claims in published maps and institutional affiliations.

\section{Author details}

${ }^{1}$ Physical Therapy Department, School of Rehabilitation Sciences, Shiraz University of Medical Sciences, Shiraz, Iran. ${ }^{2}$ Rehabilitation Sciences Research Center, Shiraz University of Medical Sciences, Shiraz, Iran. ${ }^{3}$ School of Rehabilitation Sciences, Shiraz University of Medical Sciences, Abiverdi 1 St,Chamran Blvd., P.O. Box 71345-1733, Shiraz, Iran.

Received: 14 July 2017 Accepted: 4 April 2018

Published online: 17 May 2018

References

1. Adams MA, Bogduk N, Burton K, Dolan P. The Biomechanics of Back Pain, 2nd Ed. Edinburgh: Churchill Livingstone; 2006.

2. Lisi AJ, Holmes EJ, Ammendolia C. High-velocity low-amplitude spinal manipulation for symptomatic lumbar disk disease: a systematic review of the literature. J Manip Physiol Ther. 2005;28:429-42.

3. Galm R, Fröhling M, Rittmeister M, Schmitt E. Sacroiliac joint dysfunction in patients with imaging-proven lumbar disc herniation. Eur Spine J. 1998;7:450-3.

4. Madani SP, Dadian M, Firouznia K, Alalawi S. Sacroiliac joint dysfunction in patients with herniated lumbar disc: a cross-sectional study. J Back Musculoskel Rehabil. 2013;26:273-8

5. Weksler N, Velan GJ, Semionov M, Gurevitch B, Klein M, Rozentsveig V, Rudich T. The role of sacroiliac joint dysfunction in the genesis of low back pain: the obvious is not always right. Arch Orthop Trauma Surg. 2007;127: 885-8.

6. Hertling D, Kessler RM. Management of common musculoskeletal disorders : physical therapy principles and methods. 4th ed. Philadelphia: Lippincott Williams \& Wilkins; 2006.

7. Harte AA, Baxter GD, Gracey JH. The efficacy of traction for back pain: a systematic review of randomized controlled trials 1, 2. Arch Phys Med Rehabil. 2003;84:1542-53.

8. Clare HA, Adams R, Maher CG. Construct validity of lumbar extension measures in McKenzie's derangement syndrome. Man Ther. 2007;12:328-34.

9. Marshall PW, Murphy BA. Evaluation of functional and neuromuscular changes after exercise rehabilitation for low back pain using a Swiss ball: a pilot study. J Manip Physiol Ther. 2006;29:550-60.

10. Liu J, Zhang S. Treatment of protrusion of lumbar intervertebral disc by pulling and turning manipulations. J Trad Chin Med. 2000;20:195.

11. Floman $Y$, Liram N, Gilai A. Spinal manipulation results in immediate H-reflex changes in patients with unilateral disc herniation. Eur Spine J. 1997;6:398-401.

12. Santilli V, Beghi E, Finucci S. Chiropractic manipulation in the treatment of acute back pain and sciatica with disc protrusion: a randomized doubleblind clinical trial of active and simulated spinal manipulations. Spine J. 2006;6:131-7.

13. Leemann S, Peterson CK, Schmid C, Anklin B, Humphreys BK. Outcomes of acute and chronic patients with magnetic resonance imaging-confirmed symptomatic lumbar disc herniations receiving high-velocity, low-amplitude, spinal manipulative therapy: a prospective observational cohort study with one-year follow-up. J Manip Physiol Ther. 2014;37:155-63.

14. Ehrler M, Peterson C, Leemann S, Schmid C, Anklin B, Humphreys BK. Symptomatic, MRI confirmed, lumbar disc Herniations: a comparison of outcomes depending on the type and anatomical axial location of the hernia in patients treated with high-velocity, low-amplitude spinal manipulation. J Manip Physiol Ther. 2016;39:192-9.

15. Khuffash B, Porter R. Cross leg pain and trunk list. Spine. 1989;14:602-3.

16. Kosteljanetz M, Bang F, Schmidt-olsen S. The clinical significance of straight-leg raising (Lasegue's sign) in the diagnosis of prolapsed lumbar disc: Interobserver variation and correlation with surgical finding. Spine. 1988;13:393-5. 
17. Aure $\mathrm{OF}$, Nilsen $\mathrm{JH}$, Vasseljen $\mathrm{O}$. Manual therapy and exercise therapy in patients with chronic low back pain: a randomized, controlled trial with 1-year follow-up. Spine. 2003;28:525-31.

18. Cleland JA, Fritz JM, Childs JD, Kulig K. Comparison of the effectiveness of three manual physical therapy techniques in a subgroup of patients with low back pain who satisfy a clinical prediction rule: study protocol of a randomized clinical trial [NCT00257998]. BMC Musculoskelet Disord. 2006;7:1.

19. Kamali F, Shokri E. The effect of two manipulative therapy techniques and their outcome in patients with sacroiliac joint syndrome. J Bodyw Mov Ther. 2012;16:29-35.

20. Childs JD, Piva SR, Fritz JM. Responsiveness of the numeric pain rating scale in patients with low back pain. Spine. 2005;30:1331-4.

21. Salaffi F, Stancati A, Silvestri CA, Ciapetti A, Grassi W. Minimal clinically important changes in chronic musculoskeletal pain intensity measured on a numerical rating scale. Eur J Pain. 2004:8:283-91.

22. Ostelo RW, de Vet HC. Clinically important outcomes in low back pain. Best Pract Res Clin Rheumatol. 2005;19:593-607.

23. Roland M, Fairbank J. The Roland-Morris disability questionnaire and the Oswestry disability questionnaire. Spine. 2000;25:3115-24.

24. Fritz JM, Irrgang JJ. A comparison of a modified Oswestry low back pain disability questionnaire and the Quebec back pain disability scale. Phys Ther. 2001:81:776-88.

25. Carmichael JP. Inter-and intra-examiner reliability of palpation for sacroiliac joint dysfunction. J Manip Physiol Ther. 1987;10:164-71.

26. Herzog W, Read L, Conway P, Shaw L, McEwen M. Reliability of motion palpation procedures to detect sacroiliac joint fixations. J Manip Physiol Ther. 1989;12:86-92.

27. Cibulka MT, Delitto A, Koldehoff RM. Changes in innominate tilt after manipulation of the sacroiliac joint in patients with low back pain: an experimental study. Phys Ther. 1988;68:1359-63.

28. Oliphant D. Safety of spinal manipulation in the treatment of lumbar disk herniations: a systematic review and risk assessment. J Manip Physiol Ther. 2004;27:197-210.

29. Magee DJ. Orthopedic physical assessment, 6th ed. St. Lois: Elsevier; 2014.

30. Levangie PK. The association between static pelvic asymmetry and low back pain. Spine. 1999;24:1234-42.

31. Cibulka MT, Rose SJ, Delitto A, Sinacore DR. Hamstring muscle strain treated by mobilizing the sacroiliac joint. Phys Ther. 1986;66:1220-3.

32. Herzog W, Scheele D, Conway PJ. Electromyographic responses of back and limb muscles associated with spinal manipulative therapy. Spine. 1999;24: $146-52$.

33. Vernon $\mathrm{H}$. Qualitative review of studies of manipulation-induced hypoalgesia. J Manip Physiol Ther. 2000;23:134-8.

34. Lehman GJ, McGill SM. The influence of a chiropractic manipulation on lumbar kinematics and electromyography during simple and complex tasks: a case study. J Manip Physiol Ther. 1999;22:576-81.

35. Cramer GD, Tuck NR, Knudsen JT, Fonda SD, Schliesser JS, Fournier JT, Patel P. Effects of side-posture positioning and side-posture adjusting on the lumbar zygapophysial joints as evaluated by magnetic resonance imaging: a before and after study with randomization. J Manip Physiol Ther. 2000;23:380-94.

36. Whittingham W, Nilsson N. Active range of motion in the cervical spine increases after spinal manipulation (toggle recoil). J Manip Physiol Ther. 2001;24:552-5.

37. Maigne J-Y, Guillon F. Highlighting of intervertebral movements and variations of intradiskal pressure during lumbar spine manipulation: a feasibility study. J Manip Physiol Ther. 2000;23:531-5.

38. Isaacs ER, Bookhout MR. Bourdillon's Spinal Manipulation. 6th ed. Boston: Butterworth Heinemann; 2002.

39. Burton AK, Tillotson KM, Cleary J. Single-blind randomised controlled trial of chemonucleolysis and manipulation in the treatment of symptomatic lumbar disc herniation. Eur Spine J. 2000:9:202-7.

40. Hahne AJ, Ford JJ, McMeeken JM. Conservative management of lumbar disc herniation with associated radiculopathy: a systematic review. Spine. 2010; 35:E488-504.

Ready to submit your research? Choose BMC and benefit from:

- fast, convenient online submission

- thorough peer review by experienced researchers in your field

- rapid publication on acceptance

- support for research data, including large and complex data types

- gold Open Access which fosters wider collaboration and increased citations

- maximum visibility for your research: over $100 \mathrm{M}$ website views per year

At BMC, research is always in progress.

Learn more biomedcentral.com/submissions 